\title{
Les Français parlent aux Français
}

\section{Frenchmen speaking to their countrymen}

\author{
Jean-Marc GUILÉ \\ Rédacteur en chef \\ guile.jean-marc@chu-amiens.fr
}

( $\mathrm{n}$ cette mi-novembre, il faut reprendre le chemin de la résistance, l'arpenter de nouveau avec un goût de sang, une odeur de gâchis et d'impuissance à la bouche. Et puis laisser le deuil entrer, nous emporter dans un grand mouvement de solidarité, nourri du sursaut national de janvier, mais bridé et tamisé aux couleurs de novembre, tout à la fois intimiste et partagé, face à la mort d'autres nous-mêmes, de proches et d'inconnus, fauchés là où nous aurions pu être nous-mêmes.

Très rapidement, des photos aux couleurs chaudes jaune-orangé, mêlant fleurs et bougies, ont couvert de leurs teintes familières et intimes, les murs des réseaux sociaux, s'intégrant à la narration du deuil national occultant ce que les premières images pouvaient comporter de crudité, de violence et d'inhumanité. Par leurs actes qui « dépassent notre entendement » comme ont pu l'exprimer des responsables politiques, les terroristes «se mettent hors humanité ». Dans le discours de nos compatriotes, « ils se mettent hors nation », se moquant bien de déchoir d'une nationalité qu'ils ont déjà fait choir avant de passer à l'acte. Ils sont exclus de l'histoire et du récit national, expulsés loin de nous, de notre humanité. Ce sont des étrangers, des migrants envieux.

Et pourtant si c'était nous, si ces terroristes étaient, pour une part au moins, comme la révélation d'un ennemi intime?

$\mathrm{Au}$ vu des réactions plus ou moins médiatisées ou politisées, les nôtres et celles de nos compatriotes, disons que nous avons à faire à des fonctionnements de groupe en identification projective pathologique. Depuis quelques décennies, nos sociétés voient leurs systèmes de sens et d'action évoluer sur fond de mondialisation. Pour reprendre les mots de Kaës (2012) l'ébranlement des garants métasociaux, portés par la Loi, la Religion et la Culture, fragilise les garants métapsychiques du fonctionnement individuel et groupal ${ }^{1}$. La société française est un enchevêtrement de multiple groupes, chaque individu pouvant appartenir à plusieurs groupes (amis, voisins, réseau social, parti politique...). Chaque groupe fonctionne comme un appareil à penser et à transformer les contenus psychiques de chacun des membres du groupe. Quand on stigmatise « l'affaiblissement de la parole politique et le silence des intellectuels », on dit la panne de ces machines groupales à transformer. Le seul exutoire de notre violence qu'on tente de dénier, n'est bien souvent que le thrill voyeuriste des chaînes d'information continue. Le fonctionnement groupal,

1. En référence au fonctionnement des groupes humains, Kaës (2012) définit au sein des groupes, plusieurs espaces psychiques emboîtés dont l'articulation est garantie par des formations métapsychiques, ellesmêmes appuyées sur des garants métasociaux. Le groupe agit comme espace de transformation des contenus psychiques des membres composant le groupe. Développant une intuition de Piera Aulagnier, Kaës décrit le contrat narcissique comme l'une des modalités d'alliance inconsciente intersubjective. Contrepartie de son investissement par ses prédécesseurs et ses pairs, le sujet est investi d'une mission de continuité, de prolongation post-mortem de ceux qui l'investissent. Cette alliance inconsciente est l'un des opérateurs des transformations psychiques groupales. 
avec ses alliances inconscientes au sens de Kaës, est à l'œuvre. Des forces médiatiques, culturelles, politiques se conjuguent pour occulter une réalité irreprésentable et déplacer la menace vers un autre, totalement autre, externalisé, peut-être plus facilement représentable mais uniquement dans ce qu'il aurait fantasmatiquement de différent de nous. Mais ce qui est dénié fait retour, dans sa brutalité, sa crudité, comme ce qui est croqué dans le dessin de Bartok: le tchak tchak tchak du bruit, de l'indifférencié, du barbare non transformé en langage, de l'envie et de la haine primitive. Dans la multiplicité des échanges intergroupaux en identification projective, les individus se renvoient de psyché en psyché leurs contenus destructeurs. Dans ce fonctionnement en identification projective pathologique, la destructivité est projetée en nous, par le biais des fonctionnements psychiques de nos groupes d'appartenance. Mais notre fragilité psychique groupale actuelle propose encore trop peu d'appareils à transformer.

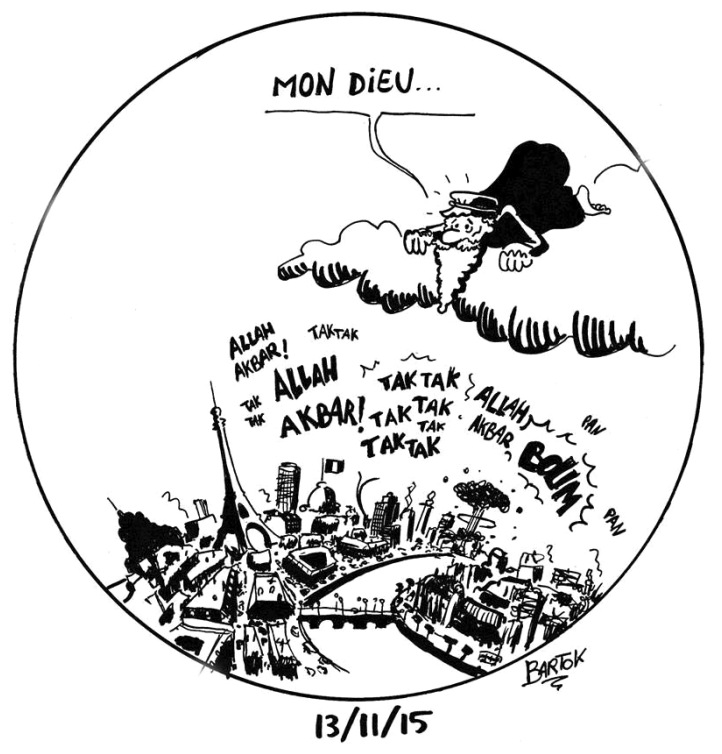

C Barthélémy Bernat.

Pour beaucoup, Internet fournit une plateforme pour recréer des mythes, proposer des narrations de groupe qui peuvent être des discours prêt-à-porter comme les thèses complotistes. Internet devient une formation métasociale contemporaine, en capacité de soutenir la formation de nouvelles alliances narcissiques de groupe. La posture apocalyptique de nos terroristes est un exutoire narcissique de l'envie primitive réveillée en

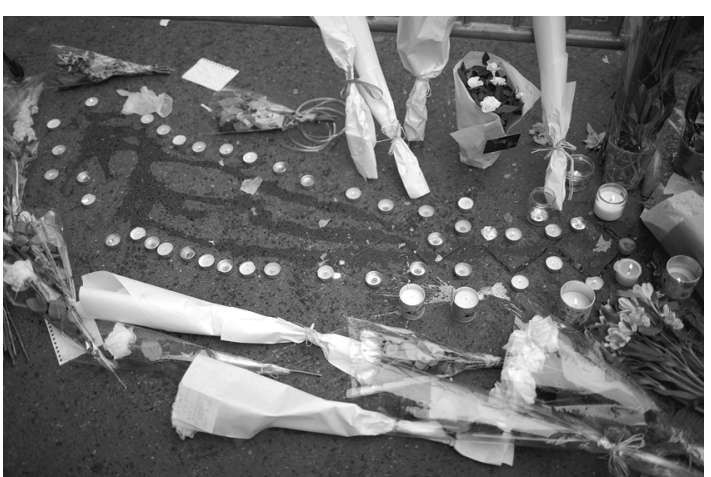

(c) Christopher Furlong/Getty Images.

eux ou bien jamais tarie. Leur mouvement, une régression totalitaire. Elle s'inscrit sur la toile de fond de la transmission, marquée actuellement par l'échec du contrat narcissique, par l'effritement de la continuité narcissique entre jeunes et aînés, entre ceux des terres oubliées de la nation et les nantis, entre les générations de désillusionnés de la République.

Quelle réponse apporter? Soit du côté des forces du moi groupal: «on continue comme avant... On se tient debout », comme l'illustre la photo de la communauté Facebook J'aime Paris aimée deux cent mille fois et partagée cent mille fois : des milliers de chaussures posées les unes à côté des autres, place de la République, malgré la pluie et l'interdiction des rassemblements. Soit et cela peut se conjuguer avec la première voie : user et abuser de la puissance de transformation culturelle, la caricature jouant du rire et du dessin, mais aussi la stylisation du sang comme l'illustre la très belle photo de Christopher Furlong qui donne à voir dans une représentation évoquant un tableau haïtien, les différentes strates d'émotions et de réponses qui ont transformé en œuvre artistique le sang répandu sur les trottoirs : à côté des gants et des tubulures laissés là par le SAMU et les pompiers, les mots sur les papiers qui doublent les gestes qui sauvent, puis les bougies qui ouvrent une dimension spirituelle, puis viennent les fleurs comme des coups de pinceaux successifs, multicolores, ininterrompus, toujours renouvelés.

\section{RÉFÉRENCE BIBLIOGRAPHIQUE}

Kaës, R. (2012) Le malêtre. Paris, Dunod. 